\title{
Implementation Mechanism of Automated Reconciliation for Web Services Protocols
}

\author{
Haidong $\mathrm{Yu}^{1,2, a}$, Ying Zou ${ }^{3}$ and Fang $\mathrm{Liu}^{1}$ \\ ${ }^{1}$ Department of Library Information Service, China Three Gorges University, Yichang, China \\ ${ }^{2}$ Department of Control Science and Engineering, Huazhong University of Science and Technology, \\ Wuhan, China \\ ${ }^{3}$ Banking Department, Three Gorges Finance Co., Ltd, Yichang, China \\ ahustyhd@163.com
}

Keywords: web service, mechanism design, interaction process reconciliation

\begin{abstract}
The paper mainly researched the game actions among partners in complex interactions supported by Web Service. It studied web service provider's implementation decision satisfied with perfect Bayesian equilibrium thus results a controllable equilibrium route in voluntary implementation. It also contributes to the problem of voluntary implementation when the planner is also a player and finds out the full characterization of it. So the implementation mechanism can always get the reconciliation of web service, which is able to effectively facilitate dynamic interactions among trading partners in a peer to peer mode.
\end{abstract}

\section{Introduction}

Heterogeneous database integrated approach has great merit which can solve the problem of heterogeneous database and synchronization mechanism in enterprise data integration, then a database centre is linked up by web service, and a transparent unified interface is provided[1]. Users can not only query the database of the center, but also add, delete, update operation, and synchronize to the source database, at the same time, the source database client data and structure changes can be synchronized to the central database. When design the methodology, an attribute-based access control model for web services with negotiation to provide an effective access control mechanism is considered prior. Usually, the model is based on SAML and XACML and takes the restrictive condition composed of identity attributes and context attributes to provide fine-grained access control, but it need negotiation ability that can make service requester communicate with the service provider and change the parameters in the request to get access to the services. So, if the Web services can be implemented volunteerly by different agents or enterprises who design or possess the component element, regulation and key technology, web services will be more efficient. For example, changing the service scheduling of service proxy from the synchronous model to the pure asynchronous model or adopting NS2 simulator to test the performance of the engine under the circumstance above as well as test the performance of the engine with service proxy and without service proxy respectively, the simulation results conclude that the access flow of the engine with service proxy is more than the flow of the engine without service proxy on the condition of the same thread resources[2-6]. Moreover decoupling the process execution, the differentiated web service scheduling and the pure asynchronous scheduling model greatly enhance the utilization efficiency of the engine, increase the concurrence of the process execution and improve the throughput and the performance of the engine.

In this paper, we mainly researched the game actions among partners in complex interactions supported by web service. It studied web service provider's implementation decision satisfied with perfect Bayesian equilibrium thus resulting a controllable equilibrium route in voluntary implementation. It also contributes to the problem of voluntary implementation when the planner is also a player and finds out the full characterization of it. 


\section{Basic Model and Equilibrium Analysis}

In this section, we examine a simple bargaining setting, where heterogeneous partners including planner in web service are repeatedly matched with each other. We begin by characterizing efficiency in such a dynamic setting, and discuss how it differs from efficiency in a centralized static setting. It takes an implementation approach, characterizing the possible reconciliation rules which result as the extensive game form is varied, particularly concerned with the impact of making interaction among partners in web service: imposing individual rationality on and off the equilibrium path of voluntary implementation.

In voluntary implementation, the outcome determined by the mechanism is a medium outcome, the relation between the medium outcome and the outcome implemented can be defined as an outcome $G: A \times T \rightarrow A$. If the state is $t$, the outcome determined by the mechanism is $a$, then the outcome implemented is $G(a, t)$.

The message space of agents is $M=\times M_{i}$, with $M_{i}$ denoting message space of agent $i$. Let $\sigma_{i}: T_{i} \rightarrow M_{i}$ be the strategy of agent $i$ and $\sigma: T \rightarrow M$. For any $m \in M$, let $\sigma^{-1}(m) \equiv\{t \in T, \sigma(t)=m\}$ be the state set that agents send $m$ when they adopt strategy $\sigma$. Let $\sigma(T) \equiv\{m \in M: m=\sigma(t)$ for some $t \in T\}$. The strategy of the planner is a function $\alpha: M \rightarrow A$.

A deception for agent $i$ is $\theta_{i}: T \rightarrow 2^{T}$, which satisfies for any $t \in T, t \in \theta_{i}(t)$. Let $\theta(t)=\bigcap_{i \in \Gamma} \theta_{i}(t)$. If agents adopt strategy $\sigma$, then for any $t \in T, \theta(t)=\sigma^{-1}(\sigma(t)), \theta_{i}(t)=\sigma_{i}^{-1}\left(\sigma_{i}(t)\right)$ and $\theta_{-i}(t)=\sigma_{-i}^{-1}\left(\sigma_{-i}(t)\right)$. For each $i \in \Gamma$, if $T_{i} \subseteq T$, then define $D\left(T_{1}, \cdots, T_{n}\right)$ as $D\left(T_{1}, \cdots, T_{n}\right) \equiv\left\{i: \bigcap_{j \neq i} T_{j} \neq \varnothing, \bigcap_{j \in \Gamma} T_{j}=\varnothing\right\}$.

Definition $\left(\sigma, \alpha, \mu^{*}\right)$ is a perfect Bayesian equilibrium (henceforth PBE) if:

(1) for any $i \in \Gamma$, any $t \in T$ and any $m_{i} \in M_{i}, G(\alpha(\sigma(t)), t) R_{i}(t) G\left(\alpha\left(\sigma_{-i}(t), m_{i}\right), t\right)$

(2) if $m \in \sigma(T)$, then $\alpha(m) \in \underset{a \in A}{\arg \max } \sum_{t \in \sigma^{-1}(m)} \mu(t) u_{0}(G(a, t), t)$

(3) if $m$ does not satisfies (2), then $\alpha(m) \in \underset{a \in A}{\arg \max } \sum_{t \in T} p(m)(t) u_{0}(G(a, t), t)$, where $p(m) \in \Delta(T)$ is arbitrary

(4) if $m$ satisfies (2), then $\mu^{*}(m)=r\left(\sigma^{-1}(m)\right)$, otherwise $\mu^{*}(m)=p(m)$.

A compromise selection $b$ is a function satisfies: for any $S \in 2^{T}$, $b(S) \in \underset{a \in A}{\arg \max } \sum_{t \in S} \mu(t) u_{0}(G(a, t), t)$.

Consider a web service interaction entry right reconciliation problem. Suppose there are $n$ $(n \geq 3)$ number of manufacturers and the military wants to select $k(n>k \geq 2)$ manufacturers to entry into the web service industry. Let $\Gamma=\{1, \cdots, n\}$ be the set of manufacturers, $t_{i}\left(0 \leq t_{i} \leq 1\right)$ be the technology level of manufacturer $i$, and $. t=\left(t_{1}, \cdots, t_{n}\right)$ be the technology level profile of manufacturers. Let $a=\left(a_{1}, \cdots, a_{n}\right)$ be an reconciliation of entry right, according to which for each $i \in \Gamma, a_{i}=1$ means manufacturer $i$ gets the entry right and $a_{i}=0$ means manufacturer $i$ is ruled out. Let $A \equiv\left\{a: \sum a_{i}=k\right\}$ be the reconciliation set of entry right, by element of which only $k$ manufacturers are allowed to entry into the web service industry. In order to ensure the quality of web service interaction, the object of the military is to select manufacturers with the top $k$ highest technology levels. Allocating entry right to manufacturers with the top $k$ highest technology levels is optimal reconciliation of web service interaction entry right. Let $F$ be the object function of the military, then for any technology level profile $t$, the optimal reconciliation of web service interaction entry right is in $F(t)$, where $F(t) \equiv\left\{a: a \in A, a \in \arg \max b \cdot t^{T}\right\}$ and $t^{T}$ is the transpose of $t$. Cause different manufacturers may have the same technology level, so for a technology level profile $t$, $F(t)$ may be not single. 
For the reconciliation problem, suppose the following assumptions hold:

(A1) Manufacturers are neutral. The expected profit a manufacturer gets is relevant to the technology profile of manufacturers. If the technology profile is $t$, the reconciliation of web service interaction entry right is $a$, then the expected profit manufacturer $i$ gets is $\pi_{i}(a, t)$, where $\pi_{i}(a, t)=0$ if $a_{i}=0$, which means if a manufacturer does note get the entry right, his expected profit is zero.

(A2) If a manufacturer gets the entry right, the expected profit it gets increase as its own technology level increases, decrease as the technology level of any other manufacturer who gets the entry right increases. So for any technology level profile $t$ and any reconciliation $a$, if $a_{i}=1$, then $\frac{\partial \pi_{i}}{\partial t_{i}}(a, t)>0$, if $a_{i}=1$ and $a_{j}=1$, where $j \neq i$, then $\frac{\partial \pi_{i}}{\partial t_{j}}(a, t)<0$.

(A3) For any technology level profile $t$, any reconciliation $a$, and any $i \neq j$, if $a_{i} t_{i}=a_{j} t_{j}$, then $\pi_{i}(a, t)=\pi_{j}(a, t)$. Thus if manufacturer $i$ and manufacturer $j$ both get the entry right and their technology levels are the same, then the expected profits they get also equals to each other.

(A4) Let $\varphi:\{1, \cdots, n\} \rightarrow\{1, \cdots, n\}$ be a map. For any $n$ dimensional vectors $v$ and $v^{\prime}$, if $v^{\prime}=\left(v_{\varphi(1)}, \cdots, v_{\varphi(n)}\right)$, then say $v$ and $v^{\prime}$ are similar to each other. For any $v$, let $P M(v)$ be the vector set similar to $v$. For any reconciliation $a \in A$ and any technology level profile $t$, define a operation $a \otimes t=\left(a_{1} t_{1}, \cdots, a_{n} t_{n}\right)$. Given two technology level profile $t$ and $t^{\prime}$, two reconciliation $a$ and $a^{\prime}$, if $a_{i} t_{i}=a_{i}^{\prime} t_{i}^{\prime}$ and $a_{-i} \otimes t_{-i} \in P M\left(a_{-i}^{\prime} \otimes t_{-i}^{\prime}\right)$ hold, then $\pi_{i}(a, t)=\pi_{i}\left(a^{\prime}, t^{\prime}\right)$. Thus, the expected profit a manufacturer gets is relevant to the technology levels of other manufacturers who get the entry right, but irrelevant to the arrangement of these technology levels.

(A3) and (A4) mean that manufacturers are anonymous, which means whether a manufacturer gets the entry right and the expected profit it gets is relevant to its technology level but other properties.

Theorem If (A1)-(A4) hold, then $g$ satisfies strong incentive compatibility.

Proof: To proof the theorem, we need to prove that for any manufacturer, when other manufacturers are telling the truth, the manufacturer does not have the incentive to lie at any technology level.

Here we prove a stronger version of incentive compatibility. We prove that at technology level profile $t$, there is no manufacturer who has the incentive to lie when other manufacturers are telling the truth.

Given a technology level profile $t$, when all manufacturers are telling the truth, the reconciliation $g_{1}(t) \in F(t)$. For any $i \in \Gamma$, if $\left[g_{1}(t)\right]_{i}=1$, then $\left[g_{2}(t)\right]_{i}=\underline{\pi}\left(t_{-i}\right)$, otherwise pay zero. For any $i \in \Gamma$ and any $t_{i}^{\prime}$

(1) if $\left[g_{1}(t)\right]_{i}=1$ and $g_{1}\left(t_{i}^{\prime}, t_{-i}\right)=1$, then

$\pi_{i}\left(g_{1}(t), t\right)-\left[g_{2}(t)\right]_{i}$

$=\pi_{i}\left(g_{1}\left(t_{i}^{\prime}, t_{-i}\right), t\right)-\underline{\pi}\left(t_{-i}\right)$

$=\pi_{i}\left(g_{1}\left(t_{i}^{\prime}, t_{-i}\right), t\right)-\left[g_{2}\left(t_{i}^{\prime}, t_{-i}\right)\right]_{i}$

(2) if $\left[g_{1}(t)\right]_{i}=1$ and $g_{1}\left(t_{i}^{\prime}, t_{-i}\right)=0$, let $a=g_{1}(t) \in F(t)$, then

$$
\begin{aligned}
& \pi_{i}\left(g_{1}(t), t\right)-\left[g_{2}(t)\right]_{i} \\
& =\pi_{i}(a, t)-\pi\left(t_{-i}\right) \\
& \geq 0=\pi_{i}\left(g_{1}\left(t_{i}^{\prime}, t_{-i}\right), t\right)-\left[g_{2}\left(t_{i}^{\prime}, t_{-i}\right)\right]_{i}
\end{aligned}
$$


(3) if $\left[g_{1}(t)\right]_{i}=0$ and $g_{1}\left(t_{i}^{\prime}, t_{-i}\right)=0$, then

$$
\pi_{i}\left(g_{1}(t), t\right)-\left[g_{2}(t)\right]_{i}=0=\pi_{i}\left(g_{1}\left(t_{i}^{\prime}, t_{-i}\right), t\right)-\left[g_{2}\left(t_{i}^{\prime}, t_{-i}\right)\right]_{i}
$$

(4) if $\left[g_{1}(t)\right]_{i}=0$ and $g_{1}\left(t_{i}^{\prime}, t_{-i}\right)=1$. Let $t_{j}$ be the $k$ th highest technology in $t$. Then

$$
\begin{aligned}
& \pi_{i}\left(g_{1}\left(t_{i}^{\prime}, t_{-i}\right), t\right)-\left[g_{2}\left(t_{i}^{\prime}, t_{-i}\right)\right]_{i} \\
& =\pi_{i}\left(g_{1}\left(t_{i}^{\prime}, t_{-i}\right), t\right)-\underline{\pi}\left(t_{-i}\right) \\
& =\pi_{i}\left(g_{1}\left(t_{i}^{\prime}, t_{-i}\right), t\right)-\pi_{j}\left(g_{1}(t), t\right) \\
& \leq \pi_{i}\left(g_{1}\left(t_{i}^{\prime}, t_{-i}\right),\left(t_{-i}, t_{j}\right)\right)-\pi_{j}\left(g_{1}(t), t\right)
\end{aligned}
$$

Because $g_{1}\left(t_{i}^{\prime}, t_{-i}\right)_{i}=g_{1}(t)_{j}=1$ and $g_{1}\left(t_{i}^{\prime}, t_{-i}\right)_{-i} \otimes t_{-i} \in P M\left(g_{1}(t)_{-j} \otimes t_{-j}\right)$, then according to (A3) and (A4), $\pi_{i}\left(g_{1}\left(t_{i}^{\prime}, t_{-i}\right), t\right)-\left[g_{2}\left(t_{i}^{\prime}, t_{-i}\right)\right]_{i} \leq \pi_{i}\left(g_{1}\left(t_{i}^{\prime}, t_{-i}\right),\left(t_{-i}, t_{j}\right)\right)-\pi_{j}\left(g_{1}(t), t\right)=0$.

So for any $i \in \Gamma$ and any $t_{i}^{\prime}, \pi_{i}\left(g_{1}(t), t\right)-\left[g_{2}(t)\right]_{i} \geq \pi_{i}\left(g_{1}\left(t_{i}^{\prime}, t_{-i}\right), t\right)-\left[g_{2}\left(t_{i}^{\prime}, t_{-i}\right)\right]_{i}$ holds.

Thus, the theorem is proofed.

\section{Conclusion}

The paper mainly researched the game actions among partners in complex interactions supported by web service. It studied web service provider's implementation decision satisfied with perfect Bayesian equilibrium thus resulting a controllable equilibrium route in voluntary implementation. It also contributes to the problem of voluntary implementation when the planner is also a player and finds out the full characterization of it. So the reconciliation mechanism can always get the reconciliation of web service, no matter whether the partners' technology levels are complete information among them or not, which is able to effectively facilitate dynamic interactions among trading partners in a peer to peer mode.

\section{Acknowledgment}

This work is supported by Philosophy and Society Science Foundation of Hubei Province Education Office under Grant No. 2009Y032, Yichang Science Foundation under Grant No.A201130409 and Science Foundation of Library and Information Committee for Academic libraries of Hubei Province under Grant No.2010ZD10.

Thanks Dr. C. Liu for his good advice and strong support in the theoretical analysis, formula deduction and practical calculation procedures.

\section{References}

[1] J. Cardoso, A.Sheth., J. Miller, et al,Quality of service for workflows and web service processes. Journal of Web Semantics. 3(2004)281-308.

[2] A. Wombacher., P. Fnkhauser., M. Bendick, Match-making for business processes based on choreographies. International Journal of Web Services. 1(2004)14-32.

[3] D. Van, W. Alst, T. Basten, Inheritance of work flows: an approach to tackling problems related to change, Theoretical Computer Science. 270(2002)125-203.

[4] K. Czajkowski, S. Fitzgerald, I. Foster, C. Kesselman, Grid informati.on services for distributed resource sharing, 10th IEEE International Symposium on High Performance Distributed Computing, IEEE Press, New York, 2001, pp. 181-184.

[5] M. O. Jackson, T. R.Palfrey, Voluntary implementation. Journal of Economic Theory, 98(2001) $1-25$.

[6] J. Dedrick, K. L. Kraemer, The impact of IT on firm and industry structure: the personal computer industry. California Management Review, 47(2005)122-142. 